\title{
Aggregated diamond nanorods, the densest and least compressible form of carbon
}

Natalia Dubrovinskaia and Leonid DubrovinskyWilson CrichtonFalko LangenhorstAsta Richter

Citation: Appl. Phys. Lett. 87, 083106 (2005); doi: 10.1063/1.2034101

View online: http://dx.doi.org/10.1063/1.2034101

View Table of Contents: http://aip.scitation.org/toc/apl/87/8

Published by the American Institute of Physics

\section{Articles you may be interested in}

Superhard nanocomposite of dense polymorphs of boron nitride: Noncarbon material has reached diamond hardness

Applied Physics Letters 90, 101912 (2007); 10.1063/1.2711277

Submicron cubic boron nitride as hard as diamond

Applied Physics Letters 106, 121901 (2015); 10.1063/1.4915253

Predicting hardness of dense $C_{3} N_{4}$ polymorphs

Applied Physics Letters 88, 101906 (2006); 10.1063/1.2182109

Cubic Form of Boron Nitride

The Journal of Chemical Physics 26, 956 (2004); 10.1063/1.1745964

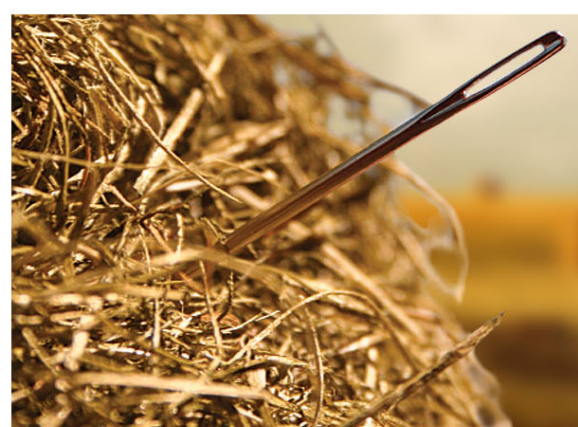

FIND THE NEEDLE IN THE
HIRING HAYSTACK

POST JOBS AND REACH THOUSANDS OF QUALIFIED SCIENTISTS EACH MONTH.

PHYSICS TODAY | JOBS

WWW.PHYSICSTODAY.ORG/JOBS 


\title{
Aggregated diamond nanorods, the densest and least compressible form of carbon
}

\author{
Natalia Dubrovinskaia ${ }^{a)}$ and Leonid Dubrovinsky \\ Bayerisches Geoinstitut, Universität Bayreuth, 95440 Bayreuth, Germany \\ Wilson Crichton \\ ESRF, B.P. 220, 38043 Grenoble Cedex, France \\ Falko Langenhorst \\ Bayerisches Geoinstitut, Universitat Bayreuth, Germany \\ Asta Richter \\ Technische Fachhochschule Wildau, Bahnofsrasse 1, 15745 Wildau, Germany
}

(Received 26 January 2005; accepted 7 July 2005; published online 16 August 2005)

\begin{abstract}
We report the synthesis of aggregated diamond nanorods (ADNRs) from fullerene $\mathrm{C}_{60}$ at 20(1) GPa and $2200{ }^{\circ} \mathrm{C}$ using a multianvil apparatus. Individual diamond nanoroads are of 5-20 nm in diameter and longer than $1 \mu \mathrm{m}$. The x-ray and measured density of ADNRs is $\sim 0.2 \%-0.4 \%$ higher than that of usual diamond. The extremely high isothermal bulk modulus $K_{T}=491$ (3) GPa [compare to $K_{T}=442$ (4) $\mathrm{GPa}$ of diamond] was obtained by in situ x-ray diffraction study. Thus, ADNRs is the densest among all carbon materials and it has the lowest so far experimentally determined compressibility. (C) 2005 American Institute of Physics. [DOI: 10.1063/1.2034101]
\end{abstract}

Due to the exciting promise for nanotechnology, nanotubes, nanowires, nanorods, and nanowhiskers have become the most attractive target for synthesis and study. ${ }^{1}$ Although production of nanowires from various materials is proving very successful, ${ }^{2,3}$ the development of diamond nanowires has been slow. So far nobody has reported experimental observation of diamond nanorods, while several theoretical groups have predicted their possible existence and tried to calculate their properties. ${ }^{4-7}$ For centuries diamond has been considered the hardest known material. ${ }^{8-10}$ However, both theory and experiment suggest that single-walled carbon nonotubes (SWNT) along their axis are stiffer and stronger than diamond. ${ }^{11,12}$ Moreover, theory predicts ${ }^{4}$ that diamond nanorod would have a brittle fracture force and a zero strain stiffness that exceeds that of carbon nonotubes for radii greater than about 1-3 $\mathrm{nm}$. The results of the theoretical study $^{7}$ indicate that diamond nanowires with the diameter from 2.7 to $9 \mathrm{~nm}$ could be stable.

Following synthesis of the first artificial diamonds in the mid 1950's, various methods of manufacturing diamonds and diamond like carbon (DLC) under variable pressure and temperature conditions have been proposed. The techniques, ranging from direct solid-state transformation of graphite under static or shock pressure to chemical-vapor deposition, allow synthesis of materials with properties approaching those of natural diamonds. ${ }^{13,14}$ Recently, the synthesis and characterization of nanometer size clusters, including nanocrystalline films of diamond-like carbon and nano- and polycrystalline cubic diamond attracts special attention. ${ }^{15-18}$ These studies are motivated by the observed ability to vary the properties of these materials according to size. ${ }^{19}$ Ultrafine diamond synthesised by explosive detonation ${ }^{14}$ was proposed to be very perspective for many traditional diamond applications such as fine polishing of ceramics and surface coating.

\footnotetext{
a) Author to whom correspondence should be addressed; electronic mail: natalia.dubrovinskaia@uni-bayreuth.de
}

Nanocrystalline forms of known materials can enhance useful properties of their macrocrystalline counterparts. Polycrystalline cubic diamond obtained by direct conversion of graphite at static high pressures and temperatures ${ }^{16}$ is ultrahard. However, it was synthesised in microquantity that was not enough for full characterization of physical properties of the sample. The first synthesis of bulk sample of nanocrystalline diamond from $\mathrm{C}_{60}$ was recently reported, ${ }^{8}$ and complex investigation has suggested that the new material was at least as hard as single crystal diamond and, at high temperature and ambient pressure, kinetically more stable with respect to graphitization than usual diamonds. The theoretical evaluations of properties of diamond nanowires and nanorods, ${ }^{4-7}$ which predicted superior properties of the latter and made them an important and viable target for synthesis, encouraged our search for the methods of their production.

We conducted a series of experiments in 5000 tonne multianvil press, using $\mathrm{C}_{60}$ as a starting material (for experimental details see Ref. 8).

The samples obtained at $20 \mathrm{GPa}$ and high temperature [2200(50) $\left.{ }^{\circ} \mathrm{C}\right]$ came out as solid cylinders (1.8 mm diameter, $3 \mathrm{~mm}$ height) of a very compact translucent material (Fig. 1). $\mathrm{X}$-ray diffraction pattern contains only the diamond-structure reflections (Fig. 2), and its Raman and IR spectra are similar to that obtained for nanodiamond. ${ }^{8}$ However, high-resolution transmission electron microscopy (HRTEM) images from the sample, slowly cooled with rate $\sim 10 \mathrm{~K} / \mathrm{min}$ after heating for about $1 \mathrm{~h}$ at $2200(50){ }^{\circ} \mathrm{C}$, show that bulk samples of diamond consist of elongated crystals, aggregated diamond nanorods (ADNRs). The crystals can be longer than $1 \mu \mathrm{m}$, whereby the needle width is only about $20 \mathrm{~nm}$ or less [Fig. $3(\mathrm{a})$. The orientation of diamond nanorods is not uniform throughout the sample, i.e., due to the orientation effect some nanorods are cut perpendicular to the long axis and therefore seem to be equiaxed nanocrystals. A bright-field image shows a closeup of the elongated crystals. The long edges of the crystals are parallel to the (111) plane and the needle axes 


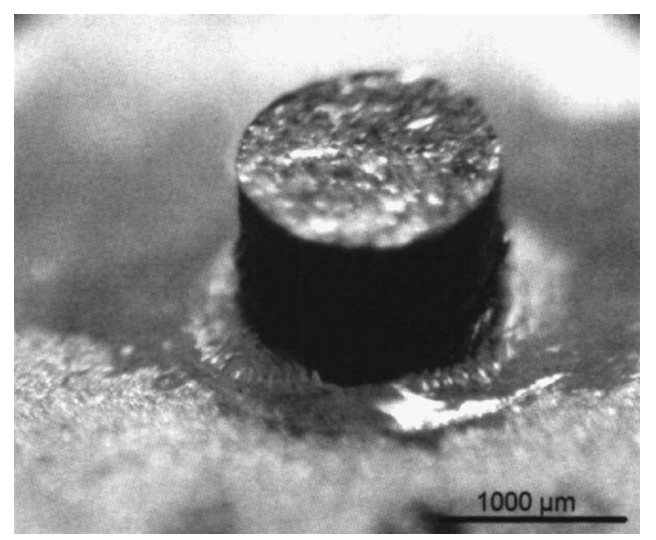

FIG. 1. (Color online). Stereo microscopy image of an ADNRs sample.

are approximately parallel $[2 \overline{1} 1]^{*}[$ Fig. $3(\mathrm{~b})]$. Due to the random orientation of nanorods it is also not possible to find a single diffraction condition, under which the rods become perfectly visible. The high hardness can possibly be attributed to this random intergrowth of diamond nanorods. The EELS spectra also correspond to diamond structure and suggest that carbon atoms near and across the numerous grain boundaries in the aggregate are also $s p^{3}$-bonded.

The $\mathrm{x}$-ray density of diamonds ${ }^{10,20}$ with natural abundances of isotopes is $3.515-3.519 \mathrm{~g} / \mathrm{cm}^{3}$. The lattice parameter of ADNRs obtained from x-ray powder diffraction is 3.5617(3) $\AA$, and consequently, the x-ray density of the material is $3.528(1) \mathrm{g} / \mathrm{cm}^{3}, \sim 0.2-0.4 \%$ higher than that of usual diamond. ${ }^{10,20}$ The samples synthesized in a multianvil apparatus have a cylindrical shape that allows us to determine their volumes. The measured density of the ADNRs bulk sample was found to be $3.532(5) \mathrm{g} / \mathrm{cm}^{3}$ in good correspondence with $x$-ray data. This result is in agreement with theoretical calculations of the structural relaxations of the

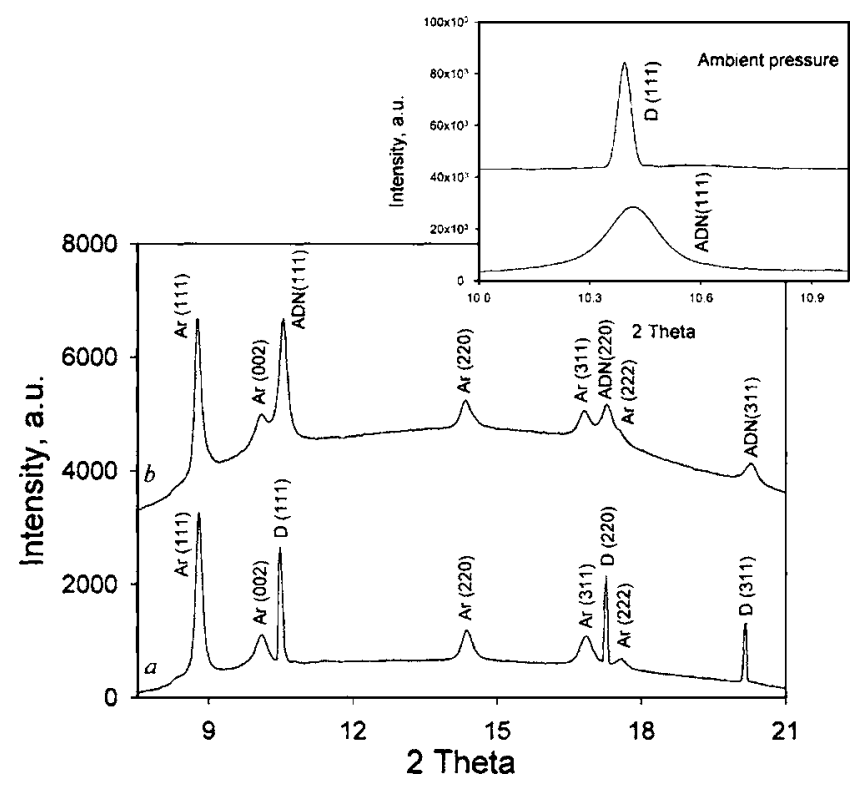

FIG. 2. Examples of diffraction patterns collected at 16.7(1) GPa from (a) diamond and (b) ADNRs compressed simultaneously in argon quasihydrostatic pressure medium. Inset shows (111) diffraction lines of diamond and ADNRs collected at ambient pressure (i.e., before cryogenic loading of $\mathrm{Ar}$ into the pressure chamber) [(D) diamond, (ADNRs) aggregate diamond nanoroads; (Ar) argon; the patterns were collected at ID30 beam line at ESRF with constant wavelength $0.3738 \AA$ ].

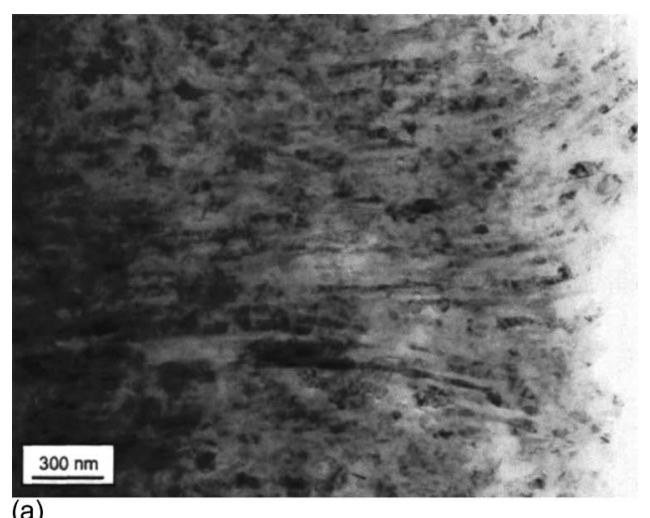

(a)

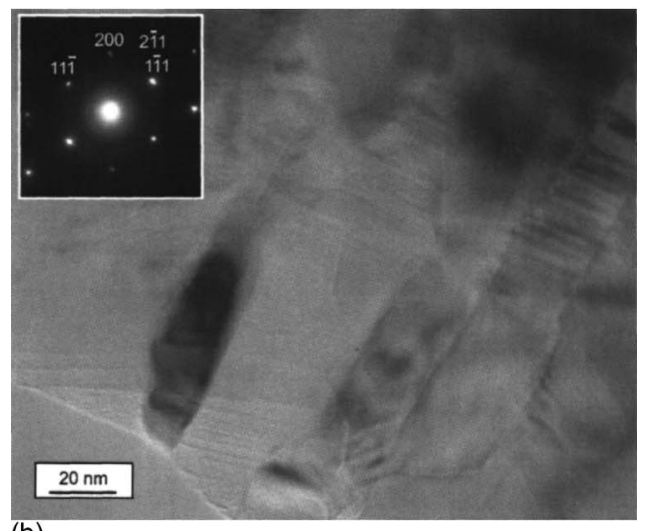

(b)

FIG. 3. (a) Bright-field TEM image of a nanocrystalline aggregate with needle-shaped, elongated crystals (diamond nanorods). The crystals can be longer than $1 \mu \mathrm{m}$, whereby the needle width is only about $20 \mathrm{~nm}$ or less; (b) bright-field image shows a closeup of the elongated crystals. The long edges of the crystals are parallel to the (111) plane and the needle axes are approximately parallel $[2 \overline{1} 1]^{*}$.

diamond nanowires. ${ }^{5}$ Although exact crystallographic configuration realized for individual rods in ADNRs was not theoretically considered in Ref. 5, the main conclusion, that the outerlayer contraction, characteristic for diamond nanowires, causes shortening of the $\mathrm{C}-\mathrm{C}$ bonds, may explain higher density of ADNRs.

In order to study the equation of state of ADNRs, isometric pieces (of about $20 \mu \mathrm{m}$ in diameter) of natural diamond and ADNRs were placed into the hole of $150 \mu \mathrm{m}$ in diameter drilled in a steel gasket indented to the thickness of $60 \mu \mathrm{m}$. Figure 3 (inset) shows the (111) diffraction lines of diamond and ADNRs collected at ambient pressure (before cryogenic loading of Ar into the pressure chamber). The diffraction line of ADNRs is evidently broader than that of diamond due to a very fine size of crystallites and it is shifted to the higher $2 \theta$ position as a consequence of the smaller lattice parameter of ADNRs. Both diamond and ANDRs were compressed simultaneously in Ar pressure medium to over $27 \mathrm{GPa}$, until the samples were bridged between diamond anvils and further compression was not possible. After high-pressure treatment x-ray diffraction pattern, Raman and IR spectra of recovered material did not change. Pressure was measured using ruby spheres of $2-3 \mu \mathrm{m}$ in diameter, placed in different parts of the sample. Figure 2 presents an example of the diffraction patterns collected at 16.7(1) GPa. Positions of the diffraction lines of Ar are the same in parts of the pressure chamber occupied by diamond and ADNRs, proving that both materials are at the same quasihydrostatic 


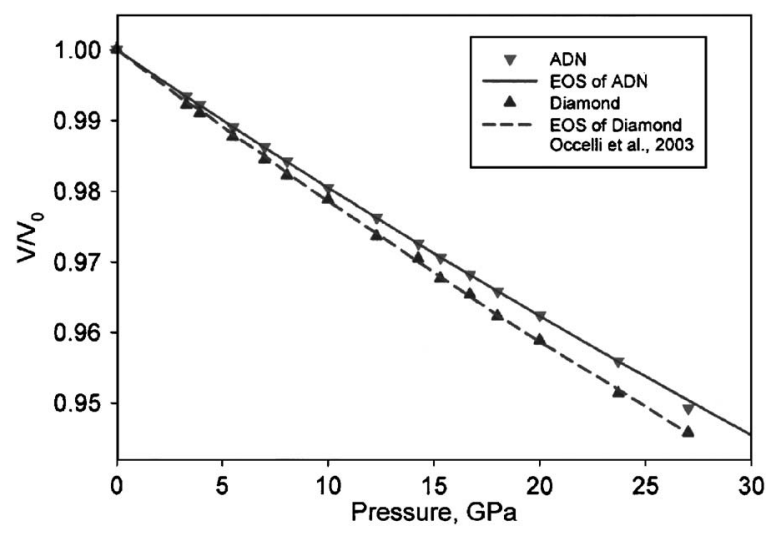

FIG. 4. (Color online). Pressure dependence of reduced volume $\left(V / V_{0}\right)$ of diamond (triangles) and aggregate diamond nanoroads (inversed triangles). Dashed line shows third-order Birch-Murnaghan equation of state (EOS) with parameters $K_{T}=446(3) \mathrm{GPa}, K^{\prime}=3.0, V_{0}=3.4170(5) \mathrm{cm}^{3} / \mathrm{mol}$ (Ref. 10). Continues line is the fit to our data with $K_{T}=491(3) \mathrm{GPa}, K^{\prime}$ $=3.1(2), V_{0}=3.4014(5) \mathrm{cm}^{3} / \mathrm{mol}$.

pressure. While the diffraction lines of diamond are very narrow, the lines of ADNRs remain broad like in the initial sample. The experimental pressure-volume data were fitted (Fig. 4) using the third order Birch-Murnaghan equation of state:

$$
\begin{aligned}
P= & 1.5 K_{T}\left[V_{o} / V\right)^{7 / 3}-\left(V_{o} / V\right)^{5 / 3}\left[1-0.75\left(4-K^{\prime}\right)\left\{\left(V_{o} / V\right)^{2 / 3}\right.\right. \\
& -1\}],
\end{aligned}
$$

(where $K_{T}, K^{\prime}, V_{o}$, and $V$ are the isothermal bulk modulus, the pressure derivative of the bulk modulus, the zeropressure volume, and the volume at a given pressure $P$, respectively). The fitting procedure for diamond give $K_{T}$ $=442(4) \mathrm{GPa}, K^{\prime}=3.2(2), V_{0}=3.4157(9) \mathrm{cm}^{3} / \mathrm{mol}$, which within the uncertainty coincide with the data from Ref. 10. For ADNRs we got $K_{T}=491(3) \mathrm{GPa}, K^{\prime}=3.1(2), V_{0}$ $=3.4014(5) \mathrm{cm}^{3} / \mathrm{mol}$. Thus, ADNRs are more than by $11 \%$ less compressible than normal diamond that makes it, an incompressible form of carbon. It has also the lowest experimentally determined compressibility ${ }^{21}$ (compare with the highest $K_{T}$ measured so far: $462 \mathrm{GPa}$ for Os, $420 \mathrm{GPa}$ for WC, $383 \mathrm{GPa}$ for Ir, $380 \mathrm{GPa}$ for cubic BN, $306 \mathrm{GPa}$ for $\mathrm{HfN})$.

Our attempts to measure microhardness of the samples failed, because a diamond tip of a Vickers-type indenter did not make indentations on the surfaces of the tested material.
The roughness of the sample was crucial for the nanoindentation experiments. The nanohardness values exceeding 100 $\mathrm{GPa}$ were obtained from flat and horizontal spots on the surface. This seems to be true, since ADNRs make clear scratches on the (111) faces of IIa natural diamond single crystals. Enhanced kinetic stability against graphitization found for nanodiamond, ${ }^{8}$ in comparison with single crystal diamond, was confirmed for ADNRs as well. We also conducted a series of mechanical tests, which suggested that under the same conditions the ADNRs material is a more effective grinding piece than normal natural or synthetic diamond, making it a potentially valuable tool for machining ferrous alloys and ceramics, and, due to its nanocrystalline nature, for precision machining. Due to its properties, the new material can be used for some nontraditional diamond applications.

${ }^{1}$ D. Appell, Nature (London) 419, 553 (2002).

${ }^{2}$ M. S. Morales and C. M. Lieber, Science 279, 208 (1998).

${ }^{3}$ M. S. Gudiksen, L. J. Lauhon, J. Wang, D. C. Smith, and C. M. Lieber, Nature (London) 415, 617 (2002).

${ }^{4}$ O. Shenderova, D. Brenner, and R. S. Ruoff, Nano Lett. 3, 805 (2003).

${ }^{5}$ A. S. Barnard, S. P. Russo, and I. K. Snook, Nano Lett. 3, 1323 (2003).

${ }^{6}$ A. S. Barnard, S. P. Russo, and I. K. Snook, Philos. Mag. 84, 899 (2004).

${ }^{7}$ A. S. Barnard and I. K. Snook, J. Chem. Phys. 120, 3817 (2004).

${ }^{8}$ N. Dubrovinskaia, L. Dubrovinsky, F. Langenhorst, S. Jacobsen, and C. Liebske, Diamond Relat. Mater., 2004, 16 (2004).

${ }^{9}$ V. Brazhkin, N. Dubrovinskaia, M. Nicol, N. Novikov, R. Riedel, V. Solozhenko, and Y. Zhao, Nat. Mater. 3, 576 (2004).

${ }^{10}$ F. Occelli, P. Loubeyre, and R. Letoullec, Nat. Mater. 2, 151 (2003).

${ }^{11}$ M. F. Yu, B. S. Files, and R. S. Ruoff, Phys. Rev. Lett. 84, 5552 (2000).

${ }^{12}$ M. Buongiorno Nardelli, B. I. Yakobson, and J. Bernholc, Phys. Rev. B 57, R4277 (1998).

${ }^{13}$ The Nature of Diamonds, edited by G. E. Harlow (Cambridge University Press, New York, 1998).

${ }^{14} \mathrm{~K}$. Xu and H. Tan, in High-Pressure Shock Compression of Solids V, edited by L. Davidson, Y. Horie, and T. Sekine (Springer, New York, 2002).

${ }^{15}$ P. Badziag, W. S. Verwoerd, W. P. Ellis, and N. R. Greiner, Nature (London) 343, 244 (1990).

${ }^{16}$ T. Irifune, A. Kurio, S. Sakamoto, T. Inoue, and H. Sumiya, Nature (London) 421, 599 (2003).

${ }^{17}$ Z. Yao, H. W. C. Postma, L. Balents, and C. Dekker, Nature (London) 402, 273 (1999).

${ }^{18}$ X. Jiang and C. L. Jia, Appl. Phys. Lett. 80, 2269 (2002).

${ }^{19}$ J. O. Orwa, S. Prawer, D. N. Jamieson, J. L. Peng, J. C. McCallum, K. W. Nugent, Y. J. Li, L. A. Bursill, and S. P. Withrow. J. Appl. Phys. 90, 3007 (2001).

${ }^{20}$ T. Yamanaka, S. Morimoto, and H. Kanda, Phys. Rev. B 49, 9341 (1994).

${ }^{21}$ H. Cynn, J. E. Klepeis, C.-S. Yoo, and D. A. Young, Phys. Rev. Lett. 88, 135701-1 (2002). 